\title{
LINC00467 facilitates osteosarcoma progression by sponging miR-217 to regulate KPNA4 expression
}

\author{
JING YAN, TAO FANG, MING ZHANG and QUAN ZHOU \\ Department of Orthopaedics, The Affiliated Huaian Hospital of Xuzhou Medical University, \\ Huaian, Jiangsu 223002, P.R. China
}

Received June 28, 2020; Accepted November 30, 2020

DOI: $10.3892 /$ ijmm.2021.4859

\begin{abstract}
Osteosarcoma (OS) is a musculoskeletal malignancy that originates from interstitial cells. An increasing number of studies have verified that long non-coding RNAs (lncRNAs) participate in the progression of numerous types of cancer. It has been reported that LINC00467 is a cancer-promoting gene in some types of cancer; however, the regulatory mechanism of LINC00467 in OS remains unknown. In the present study, reverse transcriptionquantitative PCR was used to determine LINC00467 expression in OS tissues and cells. Additionally, the impact of LINC00467-knockdown on OS cell proliferation, migration and invasion was analyzed using Cell Counting Kit-8, colony formation and Transwell assays, as well as western blot analysis. RNA pulldown and luciferase reporter assays were conducted to investigate the regulatory mechanism of LINC00467 in OS. The results delineated that LINC00467 expression was elevated in OS tissues and cells, and that high LINC00467 expression was associated with a poor prognosis in patients with OS. LINC00467 inhibition suppressed OS progression by inhibiting cell proliferation, migration, invasion and epithelial-mesenchymal transition. LINC00467 served as a molecular sponge for microRNA (miR)-217, while karyopherin subunit $\alpha 4$ (KPNA4) was a downstream target gene of miR-217. Moreover, the overexpression of KPNA4 reversed the inhibitory effects of LINC00467 inhibition on OS progression. Therefore, the present study elucidated the potential mechanism of LINC00467 in OS and indicated that LINC00467 exerted its carcinogenic effects on OS through the miR-217/KPNA4 axis, implying that LINC00467 may be a novel potential therapeutic target for OS.
\end{abstract}

Correspondence to: Dr Quan Zhou, Department of Orthopaedics, The Affiliated Huaian Hospital of Xuzhou Medical University, 62 Huaihai South Road, Huaian, Jiangsu 223002, P.R. China

E-mail:wuque197928@126.com

Key words: LINC00467, osteosarcoma, microRNA-217, karyopherin subunit $\alpha 4$

\section{Introduction}

Osteosarcoma (OS) is a highly aggressive malignant bone cancer that primarily affects children and adolescents (1). Osteosarcoma accounts for about 3-4\% of all pediatric tumors and $30 \%$ of malignant bone tumors (2). It often appears in the long bones of limbs and in the growth plate of a metaphysis (3). Several risk factors, including alkylating agents, ionizing radiation and hereditary retinoblastoma, are reported to contribute to the occurrence of OS (4). Currently, surgery, chemotherapy, immunotherapy and gene therapy are the main treatments for patients with OS; however, despite the relative effectiveness of these methods, the mortality and distant metastasis rates remain extremely high (5-7). Overall, 20-30\% of patients present with metastatic osteosarcoma, most commonly to the lungs, lymph nodes or other bones (8). The 5-year survival rate of patients with OS without any local or distant metastasis is $60-70 \%$, whereas the 5-year survival rate of patients with OS with metastasis is markedly low $(<30 \%)(9)$. Therefore, it is of great significance to fully explore the molecular mechanisms for the development of OS to develop promising treatment strategies and improve clinical outcomes.

Long non-coding RNAs (lncRNAs) are a novel class of protein non-coding transcripts that are $>200$ nucleotides in length (10). Increasing evidence has revealed that lncRNAs serve important roles in the regulation of various types of cancer $(11,12)$. For example, IncRNA SNHG5 accelerates OS progression by sponging microRNA (miRNA/miR)-212-3p and regulating serum and glucocorticoid regulated kinase 3 (13). Through the activation of the phosphatidylinositol 3-kinase/AKT signaling pathway, lncRNA SNHG12 accelerates gastric cancer progression (14). 1ncRNA EPIC1 modulates the cell cycle and promotes the proliferation of pancreatic cancer cells by interacting with Yes-associated protein-1 (15). Emerging evidence has revealed that lncRNA LINC00467 functions as a tumor-promoting gene involved in various types of cancer. For example, LINC00467 accelerates lung adenocarcinoma cell proliferation via the miR-20b-5p/cyclin D1 (CCND1) axis (16) and facilitates cervical cancer tumorigenesis by sponging miR-107 (17). However, the biological function and molecular regulatory mechanism of LINC00467 in OS remain unclear. Therefore, the purpose of the present study was to investigate the role of LINC00467 in OS progression. 


\section{Materials and methods}

Cell lines. The human normal osteoblast Hfob1.19 cell line and human OS cell lines (Saos2, MG63, U2OS and HOS) were obtained from The Cell Bank of Type Culture Collection of the Chinese Academy of Sciences. The cells were cultivated in Dulbecco's modified Eagle's medium (DMEM) supplemented with fetal bovine serum (FBS) (both Gibco; Thermo Fisher Scientific, Inc.), $100 \mathrm{U} / \mathrm{ml}$ penicillin and $100 \mu \mathrm{g} / \mathrm{ml}$ streptomycin (both Sigma-Aldrich; Merck $\mathrm{KGaA}$ ) and maintained in a humidified incubator at $37^{\circ} \mathrm{C}$ with $5 \% \mathrm{CO}_{2}$.

Tissue samples. A total of 36 paired OS and adjacent normal tissues ( $>3 \mathrm{~cm}$ from the tumor tissue) were collected from patients with OS (15 males and 21 females; age range, 26-57 years; mean age \pm SD, $47.23 \pm 4.58$ years) at The Affiliated Huaian Hospital of Xuzhou Medical University (Huai'an, China) between May 2012 and July 2014. The collected tissues were immediately frozen in liquid nitrogen and stored at $-80^{\circ} \mathrm{C}$ for subsequent experiments. None of the patients had received any preoperative anticancer treatment. The study protocol was approved by the Ethics Committee of The Affiliated Huai'an Hospital of Xuzhou Medical University. Written informed consent was obtained from all patients.

Transfection. To downregulate LINC00467 expression in cells, short hairpin RNAs (shRNAs) specifically targeting LINC00467 (sh-LINC00467\#1, 5'-GACTCATGAAACCAA TCTTCA-3'; sh-LINC00467\#2, 5'-GATGCTCTGTAAACC ACATAA-3') and a scrambled control shRNA (sh-NC, 5'-CAA CAAGATGAAGAGCACCAA-3') were designed and synthesized by Shanghai GenePharma Co., Ltd. The sequences of karyopherin subunit $\alpha 4$ (KPNA4) (Data S1) were synthesized and subcloned into pcDNA3.1 (Invitrogen; Thermo Fisher Scientific, Inc.) plasmids to generate pcDNA3.1/KPNA4. The pcDNA3.1 vector (Invitrogen; Thermo Fisher Scientific, Inc.) carrying the LINC00467 sequence (hereafter LINC00467) was used to overexpress LINC00467. An empty vector was used as a negative control. The miR-217 mimic, miR-217 inhibitor and corresponding negative non-targeting controls (NC mimics, 5'-UUCUCCGAACGUGUCACGUTT-3; miR-217 mimics, 5'-UACUGCAUCAGGAACUGACUGG-3'; NC inhibitor, 5'-UAGAACUUGCAUUGCAACCGG-3'; miR-217 inhibitor, 5'-CCAGUCAGUUCCUGAUGCAGUA-3') were purchased from Shanghai GenePharma Co., Ltd. All plasmids were transfected into U2OS and HOS cells using Lipofectamine ${ }^{\circledR}$ 2000 (Invitrogen; Thermo Fisher Scientific, Inc.) according to the manufacturer's protocol. Briefly, 100 pmol plasmids (final concentration, $50 \mathrm{nM}$ ) were diluted in $250 \mu \mathrm{l}$ serum-free Opti-MEM (cat. no. 51985042; Gibco; Thermo Fisher Scientific, Inc.) and cultured at room temperature for $5 \mathrm{~min}$. Meanwhile, another $5 \mu \mathrm{l}$ Lipofectamine 2000 was diluted in $250 \mu \mathrm{l}$ serum-free Opti-MEM and incubated for $5 \mathrm{~min}$ at room temperature. These two solutions were mixed and incubated at room temperature for $20 \mathrm{~min}$ before they were added to the cell culture wells. The transfection effects were assessed by reverse transcription-quantitative PCR (RT-qPCR) after transfection for $24 \mathrm{~h}$. The cells were then subjected to further functional assays.
Cell Counting Kit-8 (CCK-8) assay. The CCK-8 assay (Dojindo Molecular Technologies, Inc.) was utilized to test cell viability at $0,24,48$ and $72 \mathrm{~h}$ after transfection. Briefly, the cells were seeded in a 96 -well culture plate $\left(2 \times 10^{4}\right.$ cells $/ \mathrm{ml}$; $100 \mu \mathrm{l} /$ well) and cultured for 0, 24, 48 and $72 \mathrm{~h}$. Subsequently, $10 \mu \mathrm{l} \mathrm{CCK}-8$ reagent was added to each well, and the cells were cultured for $2 \mathrm{~h}$ at $37^{\circ} \mathrm{C}$. The absorbance values were measured at $450 \mathrm{~nm}$ on a microplate reader (EL340; BioTek Instruments, Inc.; Agilent Technologies, Inc.).

Colony formation assay. The transfected U2OS and HOS cells were plated in a 6-well plate and maintained in culture medium, which was replaced every 2 days for $\sim 2$ weeks. Proliferating colonies were fixed with $10 \%$ methanol for $30 \mathrm{~min}$ at room temperature and stained with $1 \%$ crystal violet (Beyotime Institute of Biotechnology) for $15 \mathrm{~min}$ at room temperature. The viable cell colonies (>50 cells) were counted and photographed under a light microscope (Olympus Corporation).

Western blot analysis. Total protein content from OS cells was extracted using RIPA lysis buffer (Beyotime Institute of Biotechnology) supplemented with phenylmethylsulfonyl fluoride. The concentration of total protein was measured using a BCA Protein assay kit (Nanjing KeyGen Biotech Co., Ltd.). The proteins (30 $\mu \mathrm{g} /$ lane) were separated via $12 \%$ SDS-PAGE and transferred to PVDF membranes. The PVDF membranes were blocked in 5\% skimmed milk for $1 \mathrm{~h}$ at room temperature and incubated with primary antibodies (Abcam) at $4^{\circ} \mathrm{C}$ overnight. After washing the membranes with $0.1 \%$ PBS-Tween 20, the membranes were further incubated with HRP-conjugated secondary antibodies (1:2,000; cat. no. ab205718; Abcam) for $2 \mathrm{~h}$ at room temperature. The protein bands were detected using an Enhanced Chemiluminescence detection system (Thermo Fisher Scientific, Inc.). GAPDH was used as the internal control. The primary antibodies were as follows: E-cadherin (1:1,000; cat. no. ab1416), N-cadherin $(1: 1,000$; cat. no. ab76011), vimentin (1:1,000; cat. no. ab92547), Slug (1:1,000; cat. no. ab51772), Twist (1:1,000; cat. no. ab50581) and GAPDH (1:2,000; cat. no. ab8245).

Transwell assays. For the migration assays, $2 \times 10^{4}$ transfected cells/well were plated in the upper chamber of Transwell inserts with $200 \mu \mathrm{l}$ serum-free DMEM. For the invasion assay, Transwell chambers (Corning, Inc.) with $8-\mu \mathrm{m}$ pores were used. The upper chamber was pre-coated with Matrigel (50 $\mu \mathrm{g} / \mathrm{ml}$; BD Biosciences) at $37^{\circ} \mathrm{C}$ for $30 \mathrm{~min}$ before the cells were plated. Next, $500 \mu 1$ DMEM with $10 \%$ FBS was added to the lower chamber. After incubation for $24 \mathrm{~h}$ at $37^{\circ} \mathrm{C}$, the cells that migrated to the lower surface of the filter were stained with $1 \%$ crystal violet for $30 \mathrm{~min}$ at room temperature. The cells were imaged using an inverted light microscope (magnification, x100; Leica Microsystems GmbH).

Subcellular fractionation location. A PARIS kit (Thermo Fisher Scientific, Inc.) was used to isolate the nuclear and cytosolic portions of OS cells according to the manufacturer's protocol. RT-qPCR assays were performed to detect the expression levels of LINC00467, GAPDH and U6 in the cytoplasm and nuclear components. GAPDH functioned as a cytoplasmic control, while U6 functioned as a nuclear control. The relative 
proportions of LINC00467, GAPDH and U6 in the cytoplasm or nucleus are shown as percentages of the total RNA.

RNA pull-down assay. The biotinylated RNAs (LINC00467 probe-biotin and LINC00467 probe no-biotin) were synthesized by Shanghai GenePharma Co., Ltd. U2OS and HOS cells were collected and lysed in specific lysis buffer containing RNase inhibitor (Beyotime Institute of Biotechnology). Subsequently, the lysate was subjected to centrifugation at $12,000 \times \mathrm{g}$ for $12 \mathrm{~min}$ at $4^{\circ} \mathrm{C}$. LINC00467 probe-biotin $(200 \mathrm{pmol})$ or LINC00467 probe no-biotin (200 pmol) was added to the supernatant and incubated for $2 \mathrm{~h}$ at $4^{\circ} \mathrm{C}$, followed by incubation with M-280 streptavidin beads (100 $\mu$; Sigma-Aldrich; Merck KGaA) pre-coated with RNase-free BSA and yeast tRNA (cat. no. TRNABAK-RO; Sigma-Aldrich; Merck $\mathrm{KGaA}$ ) for $1 \mathrm{~h}$ at $4^{\circ} \mathrm{C}$. The beads were subsequently washed twice with cold lysis buffer, three times with low-salt buffer and once with high-salt buffer. After purification using TRIzol ${ }^{\circledR}$ (Invitrogen; Thermo Fisher Scientific, Inc.), the enrichment of miRNAs was determined by RT-qPCR.

$R T$ - $q P C R$. TRIzol ${ }^{\circledR}$ reagent was used to extract the total RNA content from OS tissues and cells. Total RNA was reverse transcribed into cDNA using a PrimeScript RT reagent kit (cat. no. RRO36A; Takara Biotechnology Co., Ltd.) according to the manufacturer's protocol. A SYBR ${ }^{\circledR}$ Premix Ex Taq ${ }^{\mathrm{TM}}$ II reagent kit (cat. no. RR820A: Takara Biotechnology Co., Ltd.) was used for qPCR with an ABI7500 real-time qPCR system (Applied Biosystems; Thermo Fisher Scientific, Inc.) under the following conditions: $95^{\circ} \mathrm{C}$ for $30 \mathrm{sec}$, followed by 45 cycles at $95^{\circ} \mathrm{C}$ for $5 \mathrm{sec}$ and $60^{\circ} \mathrm{C}$ for $30 \mathrm{sec}$. U6 was regarded as the internal reference for miR-217. GAPDH was used as an internal reference for LINC00467 and KPNA4. Relative quantification was analyzed using the $2^{-\Delta \Delta \mathrm{Cq}}$ method (18). The primers used were as follows: LINC00467 forward, 5'-CCT TCTTCCTCATCATCGTC-3' and reverse, 5'-CCCAGTTTC AGTCCCTCTTG-3'; miR-217 forward, 5'-CGCTCTACT GCATCAGGAACTGA-3' and reverse, 5'-GTGCAGGGTCCG AGGT-3'; KPNA4 forward, 5'-TGTGAGCAAGCAGTGTGG GCA-3' and reverse, 5'-TGGTGGTGGGTCTTTGTGGCG-3'; GAPDH forward, 5'-CCCACTCCTCCACCTTTGAC-3' and reverse, 5'-GGATCTCGCTCCTGGAAGATG-3'; U6 forward, 5'-GCUUCGGCAGCACAUAUACUAAAAU-3' and reverse, 5'-CGCUUCACGAAUUUGCGUGUCAU-3'.

Luciferase reporter assay. The miRNAs with potential binding sites for LINC00467 were predicted using the starBase website (http://starbase.sysu.edu.cn/). Fragments of the wild-type (Wt) and mutant (Mut) reporters of LINC00467 (LINC00467-Wt and LINC00467-Mut) and KPNA4 (KPNA4-Wt and KPNA4-Mut) sharing putative binding sites with miR-217 were synthesized by Shanghai GenePharma Co., Ltd., and cloned into pcDNA3.1 vectors (Invitrogen; Thermo Fisher Scientific, Inc.). For the luciferase assay, Wt or Mut reporters were co-transfected with the miR-217 mimic or NC mimic into U2OS and HOS cells, as aforementioned. After $48 \mathrm{~h}$ of transfection, luciferase activity, as the ratio of firefly luciferase activity and Renilla luciferase activity, was determined using a Dual Luciferase Reporter Assay kit (Promega Corporation).
The sequence information of LINC00467-Wt/Mut and KPNA4-Wt/Mut is shown in Data S1.

Statistical analysis. SPSS 20.0 software (IBM Corp.) was used for data analysis. The differences among groups were compared via paired or unpaired Student's t-test, and one-way ANOVA followed by Tukey's post-hoc test. Spearman's correlation analysis was used to analyze the correlation among target genes. The survival rate was evaluated using Kaplan-Meier analysis and compared using the log-rank test. All variables are shown as the mean \pm SD. All experiments were repeated $\geq 3$ times. $\mathrm{P}<0.05$ was considered to indicate a statistically significant difference.

\section{Results}

LINC00467 expression is elevated in OS tissues and cells, and high LINC00467 expression is associated with a poor prognosis. To confirm the function of LINC00467 in OS, the expression levels of LINC00467 were examined in OS and adjacent normal tissue samples ( $n=36)$. LINC00467 expression was upregulated in OS tissues compared with in adjacent normal tissues as shown by RT-qPCR (Fig. 1A). In addition, LINC00467 expression was quantified in the four OS cell lines (Saos2, MG63, U2OS and HOS) and in normal osteoblast cells (Hfob1.19). The results revealed that LINC00467 expression was significantly upregulated in the four OS cell lines compared with in Hfob1.19 cells (Fig. 1B). Additionally, according to the median value of LIC00467 expression in OS, patients with OS were equally assigned to the low LINC00467 expression group or the high LINC00467 expression group to evaluate the clinical value of LINC00467. Notably, the overall survival time of patients with OS with high LINC00467 expression was shorter than that of patients with low LINC00467 expression (Fig. 1C). These results indicated that LINC00467 expression was elevated in OS tissues and cells, and that LINC00467 was closely associated with an adverse prognosis.

LINC00467 inhibition represses OS cell viability, migration and invasion. Having examined LINC00467 upregulation in OS, the function of LINC00467 in OS progression was explored. Considering that the U2OS and HOS cell lines presented higher LINC00467 expression than that in the other two OS cell lines, U2OS and HOS cells were selected for follow-up experiments and were transfected with shRNAs against LINC00467 (sh-LINC00467\#1 or sh-LINC00467\#2) or a negative control shRNA (sh-NC). LINC00467 expression was significantly knocked down in U2OS and HOS cells after transfection with sh-LINC00467\#1/2, especially with sh-LINC00467\#1 (Fig. 2A). Subsequently, a CCK-8 assay was performed to assess the influence of LINC00467-knockdown on OS cell viability. Compared with the sh-NC group, sh-LINC00467\#1 transfection significantly decreased the viability of U2OS and HOS cells (Fig. 2B). Colony formation assays revealed that LINC00467-knockdown triggered a significant decrease in the colony number in U2OS and HOS cells (Fig. 2C). In addition, Transwell assays revealed that LINC00467 deficiency significantly inhibited the migration and invasion of U2OS and HOS cells (Fig. 2D and E). Moreover, further experiments suggested that the protein expression 



Figure 1. LINC00467 is highly expressed in OS tissues and cells and is associated with a poor prognosis. Reverse transcription-quantitative PCR was used to detect the expression levels of LINC00467 in (A) OS and adjacent normal tissues ( $\mathrm{n}=36$ ) and (B) four OS cell lines (Saos2, MG63, U2OS and HOS) and normal osteoblast cells (Hfob1.19). (C) Kaplan-Meier analysis was performed to analyze the overall survival of patients with OS based on LINC00467 expression. The data are expressed as the mean \pm SD. Paired t-test was used for comparison between two groups, and one-way ANOVA followed by Tukey's post-hoc test for comparisons among multiple groups. $\mathrm{P}<0.05$ vs. Hfob1.19. OS, osteosarcoma.

levels of epithelial-mesenchymal transition (EMT)-associated transcription factors (Slug and Twist), as well as mesenchymal markers (N-cadherin and Vimentin), were markedly decreased by silencing LINC00467 (Fig. 2F). However, the expression levels of the epithelial marker E-cadherin were markedly upregulated in sh-LINC00467\#1-transfected cells compared with in sh-NC-transfected cells (Fig. 2F). Overall, these findings suggested that LINC00467 inhibition restrained OS progression by inhibiting cell viability, migration and invasion.

LINC00467 serves as a molecular sponge for miR-217 in OS. To study the LINC00467-mediated regulatory mechanism involved in OS progression, a subcellular fractionation location assay was implemented to confirm the location of LINC00467 in OS cells. The results delineated that LINC00467 was mainly distributed in the cytoplasm of OS cells (Fig. 3A). Subsequently, the candidate miRNAs (miR-217, miR-92b-3p, miR-197-3p, miR-485-5p, miR-1247-5p, miR-299-5p and miR-197-3p) that may be associated with LINC00467 were predicted using the starBase website. In the LINC00467 probe-biotin group, miR-217 exhibited the most enrichment compared with other miRNAs (Fig. 3B). RT-qPCR was performed to examine miR-217 expression, and the results revealed that miR-217 expression was significantly downregulated in OS tissues compared with that in adjacent normal bone tissues (Fig. 3C). Subsequently, Spearman's correlation analysis confirmed the negative correlation between LINC00467 and miR-217 expression in OS tissues (Fig. 3D). Consistently, miR-217 expression was significantly decreased in OS cell lines (Saos2, MG63, U2OS and HOS) compared with that in normal osteoblast Hfob1.19 cells (Fig. 3E). Transfection with miR-217 mimics was used to overexpress miR-217 in U2OS and HOS cells (Fig. 3F). Additionally, the effect of miR-217 overexpression was investigated on LINC00467 expression, revealing through RT-qPCR that the miR-217 mimic significantly decreased LINC00467 expression, while LINC00467 expression was significantly increased by the miR-217 inhibitor (Fig. 3G). The knockdown efficiency of the miR-217 inhibitor in cells was validated by RT-qPCR (Fig. S1A). Furthermore, the binding capacity between LINC00467 and miR-217 was analyzed. A luciferase reporter assay demonstrated that the luciferase activity of LINC00467-Wt reporters was significantly decreased in the miR-217 mimic-transfected cells; however, no significant difference was observed in luciferase activity among LINC00467-Mut groups (Fig. 3H). Overall, these findings implied that LINC00467 served as a molecular sponge for miR-217 in OS.

KPNA4 is a downstream target gene of miR-217. miRNAs have been reported to elicit their influence on cancer progression by targeting specific genes $(19,20)$. To determine the potential targets of miR-217, the underlying genes were analyzed using the starBase website (search parameters, PicTar, PITA and RNA22), identifying 7 potential mRNAs (KPNA4, SLC30A7, AP3M1, CHD9, DOCK4, EHMT1 and APAF1) using a Venn diagram (Fig. 4A). Compared with the expression levels of other candidate mRNAs, KPNA4 in U2OS and HOS cells exhibited the lowest expression after overexpression of miR-217 (Fig. 4B). Therefore, KPNA4 was selected from candidate mRNAs for subsequent investigations. KPNA4 expression was significantly elevated in the OS tissue samples compared with that in adjacent normal tissues (Fig. 4C). In addition, a negative correlation between KPNA4 and miR-27 expression, as well as a positive correlation between KPNA4 and LINC00467 expression, was observed by Spearman's correlation analysis (Fig. 4D). Moreover, the overexpression of LINC00467 significantly increased KPNA4 expression, while the miR-217 mimic significantly decreased KPNA4 expression (Fig. 4E). The overexpression efficiency of LINC00467 in cells was validated by RT-qPCR (Fig. S1B). As illustrated in Fig. 4F, only the luciferase activity of the KPNA4-Wt reporters was significantly decreased by the miR-217 mimic, confirming that KPNA4 was the downstream target gene of miR-217 and that they could bind with each other. In summary, the current findings validated that KPNA4 mRNA was a direct target of miR-217 in OS 

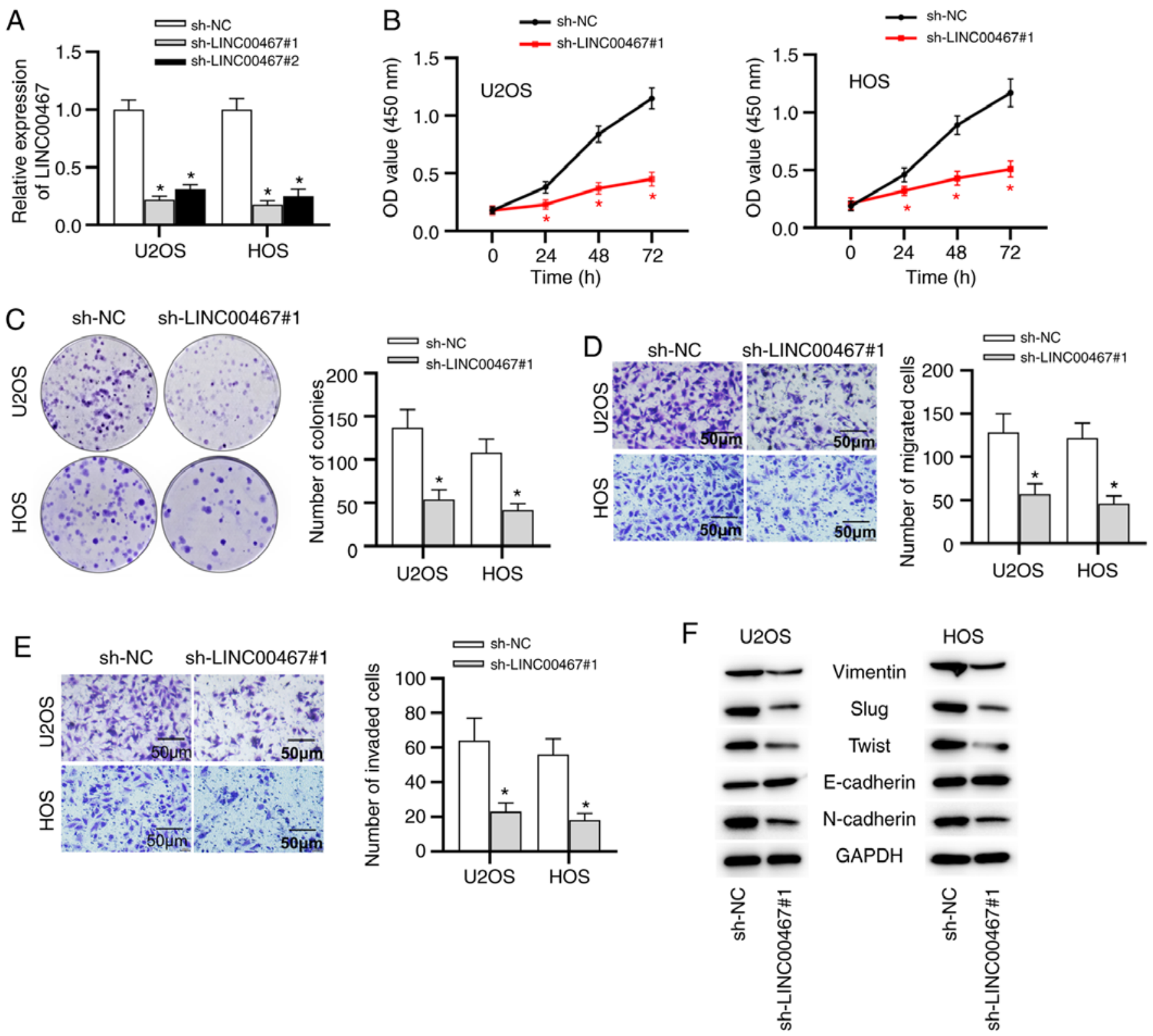

Figure 2. LINC00467 deficiency inhibits osteosarcoma cell viability, migration and invasion. (A) Reverse transcription-quantitative PCR was used to examine the knockdown efficiency of LINC00467 in U2OS and HOS cells using two shRNAs. Cell viability and the proliferative ability of each group were respectively detected by (B) Cell Counting Kit-8 and (C) colony formation assays. (D) Cell migration and (E) invasion were detected by Transwell assays. (F) Expression levels of epithelial-mesenchymal transition-associated proteins were examined by western blot analysis. The data are expressed as the mean \pm SD. Unpaired t-test was used for comparison between two groups, and one-way ANOVA followed by Tukey's post-hoc test for comparisons among multiple groups. " $\mathrm{P}<0.05$. sh, short hairpin; NC, negative control; OD, optical density.

LINC00467 promotes OS progression by upregulating KPNA4 . Finally, the present study investigated whether LINC00467 could accelerate OS progression via the miR-217/KPNA4 axis. The results revealed that KPNA4 expression was upregulated in pcDNA3.1/KPNA4-transfected cells (Fig. 5A). Additionally, transfection of pcDNA3.1/KPNA4 reversed the inhibitory role of sh-LINC00467\#1 on the viability, migration and invasion of OS cells (Fig. 5B-E). Moreover, compared with sh-LINC00467\#1-transfected cells, the protein expression levels of EMT-associated transcription factors (Slug and Twist), as well as mesenchymal markers (N-cadherin and Vimentin), were increased in the sh-LINC00467\#1 and pcDNA3.1/KPNA4 co-transfected cells (Fig. 5F). Furthermore, data from western blot analysis suggested that KPNA4 overexpression countervailed the LINC00467-knockdown-mediated inhibitory effect on the expression levels of the epithelial marker E-cadherin (Fig. 5F). Overall, the current data revealed that LINC00467 facilitated OS progression by targeting the miR-217/KPNA4 axis.

\section{Discussion}

To date, numerous studies have revealed abnormal expression levels of lncRNAs in OS (21-23). The dysregulation of lncRNAs has been verified to strongly affect the progression of OS by acting as either tumor-suppressive or oncogenic genes (23-25). Hence, identifying the specific functions of lncRNAs in OS is urgently required to validate promising 
A
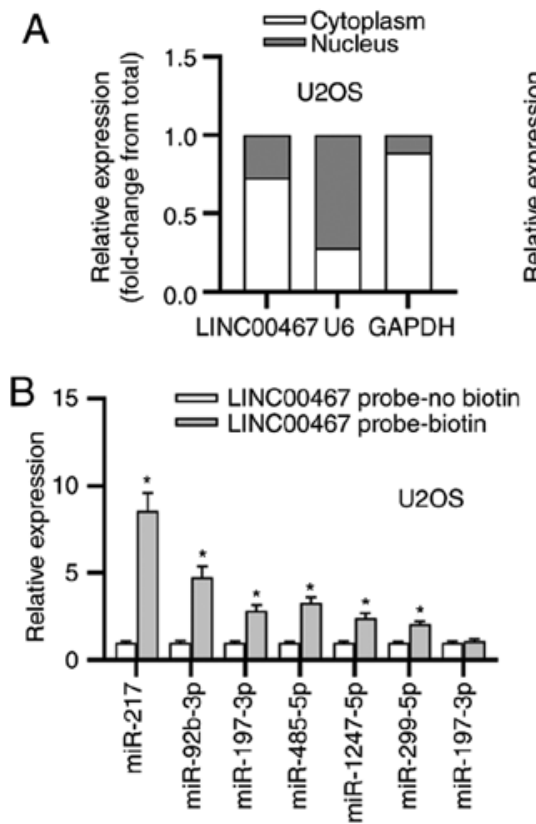

C



$\mathrm{D}$


E


$\mathrm{H}$


Figure 3. LINC00467 serves as a molecular sponge for miR-217 in OS. (A) A subcellular fractionation location assay was used to determine the distribution of LINC00467. (B) starBase website predicted the potential miRNAs of LINC00467. The binding capacity between LINC00467 and candidate miRNAs was detected by RNA pull-down assay. The data were analyzed using unpaired t-test. (C) Expression levels of miR-217 in tumor and adjacent normal tissues were confirmed by RT-qPCR. The data were analyzed using paired t-test. (D) RT-qPCR was implemented to detect the correlation between LINC00467 and miR-217 expression. (E) miR-217 expression in OS cell lines was measured by RT-qPCR. The data were analyzed using one-way ANOVA followed by Tukey's post-hoc test ("P<0.05 vs. Hfob1.19). (F) Overexpression efficiency of miR-217. (G) RT-qPCR was utilized to detect the effect of miR-217 overexpression or inhibition on LINC00467 expression. (H) Luciferase reporter assay was applied to confirm whether LINC00467 could bind to miR-217. The data were analyzed using unpaired t-test. All data are expressed as the mean \pm SD. " $\mathrm{P}<0.05$. OS, osteosarcoma; miR, microRNA; NC, negative control; RT-qPCR, reverse transcription-quantitative PCR; Wt, wild-type; Mut, mutant. 
A

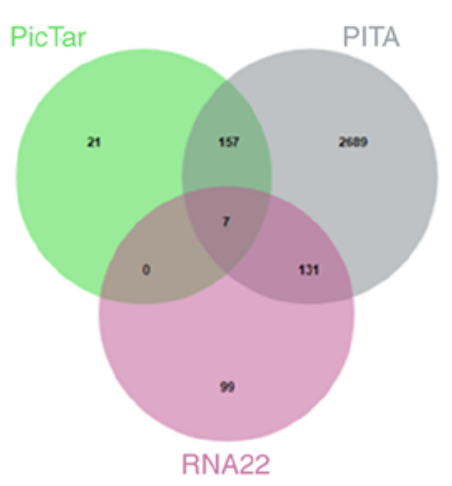

B

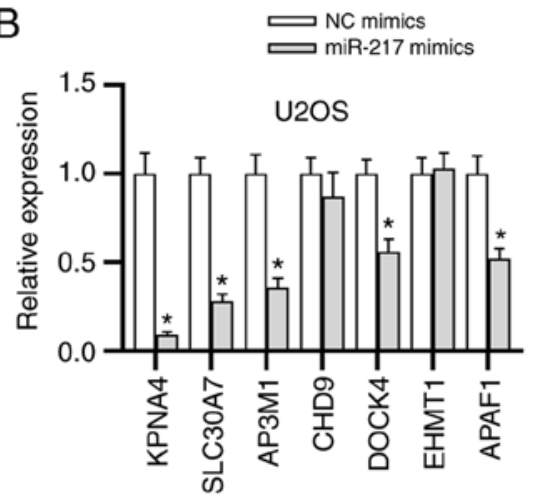



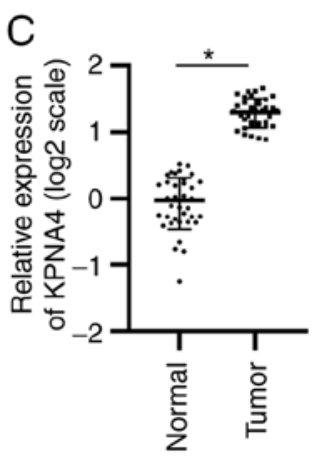

E

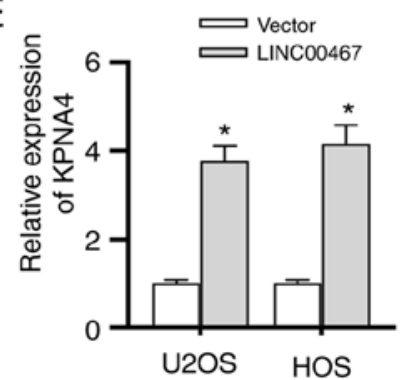

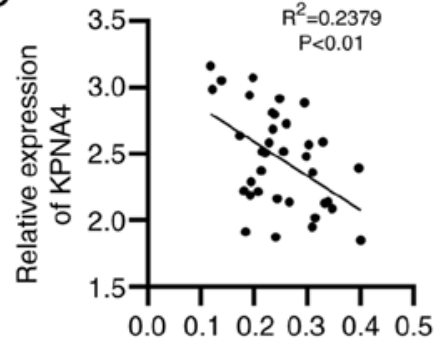

Relative expression of $\mathrm{miR}-217$



Relative expression of LINC00467

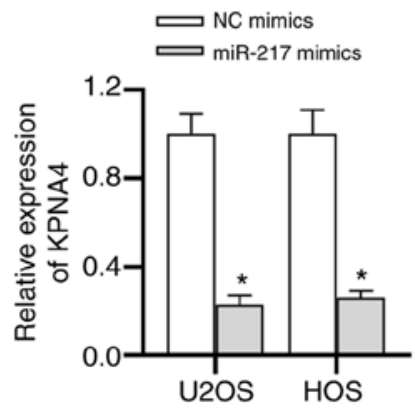

$\mathrm{F}$

KPNA4-Wt: 5' ucaAAAUAGACUGUGUUGCAGUa $3^{\prime}$ ||:||: || |||||| miR-217: 3 ' aggUUAGUCAAGGACUACGUCAU 5' KPNA4-Mut: $5^{\prime}$ ucaUUAGUCACGGACUACGUCAa $3^{\prime}$


Figure 4. KPNA4 is a downstream target gene of miR-217. (A) The starBase website (search parameters, PicTar, PITA and RNA22) predicted the potential mRNAs presented by a Venn diagram for miR-217. (B) RT-qPCR was used to detect the effect of miR-217 overexpression on the expression levels of different mRNAs. (C) Expression levels of KPNA4 in tumor and adjacent normal tissues were confirmed by RT-qPCR. (D) Spearman's correlation analysis was utilized to analyze the correlation between KPNA4 and LINC00467 or miR-217 expression. (E) RT-qPCR was applied to detect the effect of LINC00467 or miR-217 overexpression on KPNA4 expression. (F) Whether miR-217 could bind to KPNA4 was determined using a luciferase reporter assay. The data are expressed as the mean $\pm \mathrm{SD}$ and analyzed using unpaired t-test. " $\mathrm{P}<0.05$. KPNA4, karyopherin subunit $\alpha 4$; miR, microRNA; NC, negative control; RT-qPCR, reverse transcription-quantitative PCR; Wt, wild-type; Mut, mutant.

diagnostic biomarkers and therapeutic targets for OS. It has been reported that LINC00467 serves as a cancer-promoting gene in some types of cancer, such as lung adenocarcinoma, colorectal cancer and hepatocellular carcinoma $(16,26,27)$. In the present study, it was revealed that LINC00467 expression was significantly elevated in OS tissues and cell lines. High LINC00467 expression was closely associated with a poor prognosis in patients with OS. Knockdown of LINC00467 repressed the viability, migration, invasion and EMT of OS cells. 
A $\rightleftharpoons$ pcDNA3.1

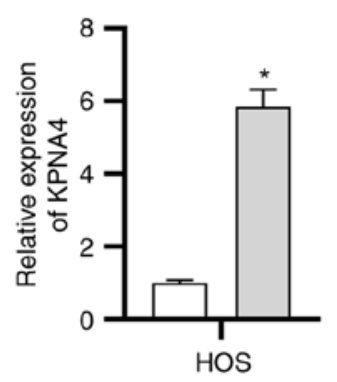

C
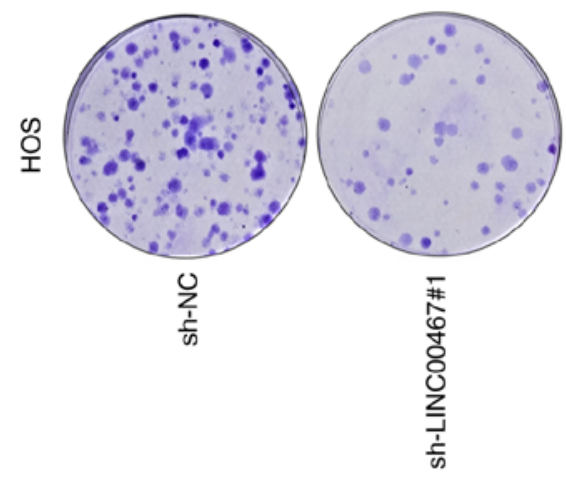

D

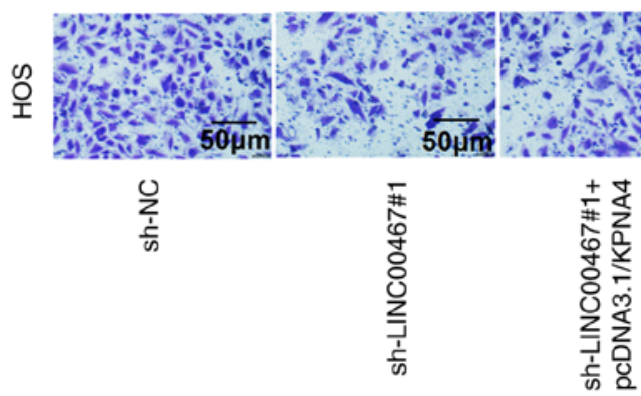



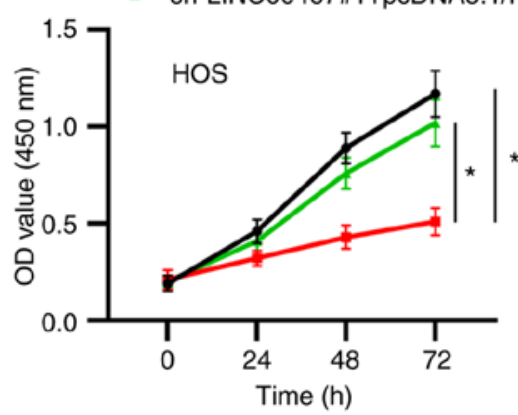

ए sh-NC

ऍ sh-LINC00467\#1

sh-LINC00467\#1+pcDNA3.1/KPNA4
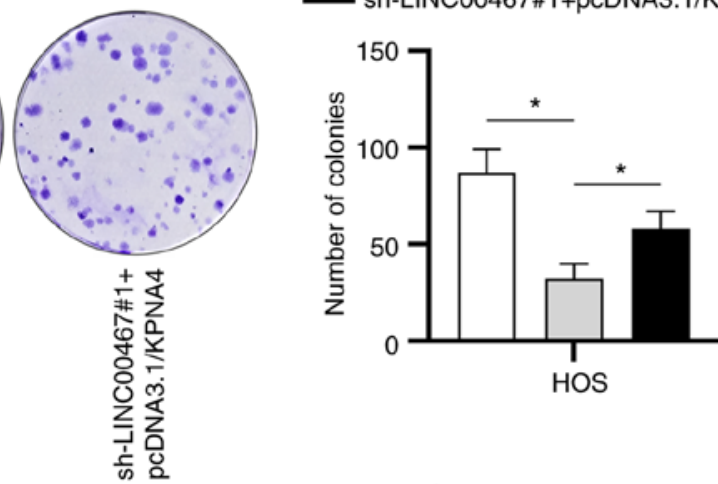

एsh-NC

ऍSh-LINC00467\#1

sh-LINC00467\#1+pcDNA3.1/KPNA4



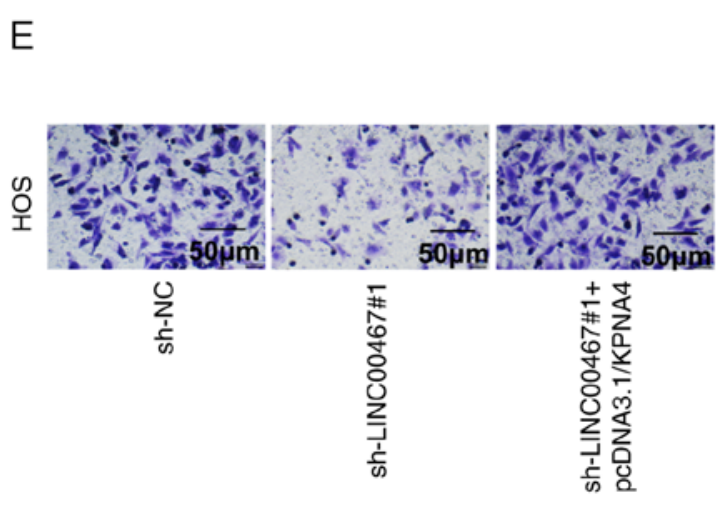

ऍ sh-NC

ए sh-LINC00467\#1

sh-LINC00467\#1+pcDNA3.1/KPNA4

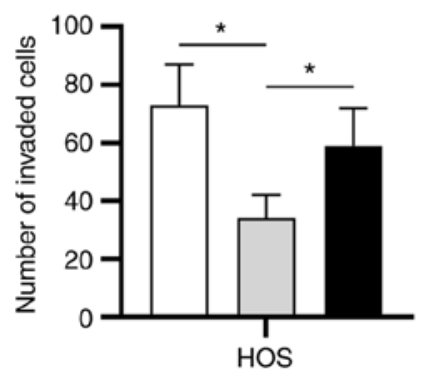

$\mathrm{F}$

HOS

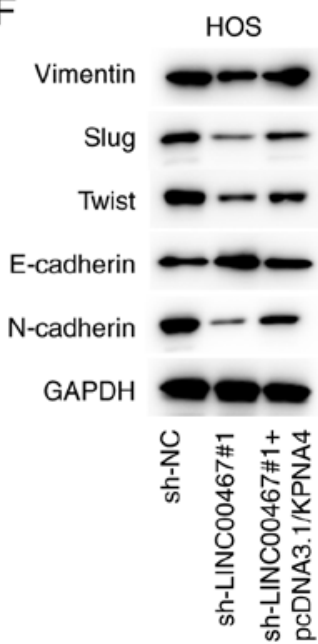

Figure 5. LINC00467 promotes osteosarcoma progression by upregulating KPNA4 expression. (A) Overexpression efficiency of KPNA4 was determined by reverse transcription-quantitative PCR. The data were analyzed using unpaired t-test. (B) Cell viability and (C) proliferation were tested by Cell Counting Kit- 8 and colony formation assays, respectively. Transwell assays were performed to evaluate (D) cell migration and (E) invasion. The data were analyzed using one-way ANOVA followed by Tukey's post-hoc test. (F) Western blot assays were used to estimate the expression levels of epithelial-mesenchymal transition-associated proteins. All data are expressed as the mean $\pm \mathrm{SD}$. ${ }^{*} \mathrm{P}<0.05$. KPNA4, karyopherin subunit $\alpha 4$; sh, short hairpin; OD, optical density; NC, negative control. 
miRNAs are short non-coding RNA molecules with 20-24 nucleotides that serve important roles in the posttranscriptional regulation of gene expression $(28,29)$. Recently, lncRNAs have been reported to serve as specific miRNA sponges to regulate various types of cancer $(30,31)$. For instance, the lncRNA LUCAT1 facilitates ovarian cancer malignancy by regulating the miR-612/human class I homeobox A13 signaling pathway (32). Through sponging miR-541-3p and thus regulating CCND1 expression, the lncRNA LOXL1-AS1 modulates prostate cancer cell proliferation and the cell cycle (33). It has been reported that LINC00467 acts as an oncogenic gene to sponge miR-20b-5p in lung adenocarcinoma (16). Nevertheless, the interaction between LINC00467 and miRNAs in OS remains unclear. In the present study, miR-217 was chosen from the potential miRNAs for LINC00467. miR-217 has been reported to have antitumor effects in multiple types of cancer $(34,35)$. For example, miR-217 inhibits EMT transition by targeting PTPN14 in gastric carcinoma (36) and suppresses HeLa cell migration and invasion by regulating MAPK1 (37). The current study validated the binding between LINC00467 and miR-217, as well as their negative correlation in OS.

It has been reported that KPNA4 exerts carcinogenic functions in human cancer (38). KPNA4 promotes angiogenesis and progression of lung cancer by knocking down MCM3AP antisense RNA 1 expression (39) and facilitates cutaneous squamous cell carcinoma tumorigenesis by inhibiting miR-3619-5p expression (40). The current study demonstrated that miR-217 could directly bind to KPNA4 and that there was a negative correlation between KPNA4 and miR-217 expression. Finally, rescue assays indicated that KPNA4 overexpression partially reversed the inhibitory effect of LINC00467 deficiency on OS progression.

In summary, the present study proved that LINC00467 silencing restrained the progression of OS by targeting the miR-217/KPNA4 axis, indicating that the LINC00467/ miR-217/KPNA4 axis may become a new biological diagnostic and therapeutic target for OS.

\section{Acknowledgements}

Not applicable.

\section{Funding}

This study was supported by the Jiangsu Natural Science Foundation (grant no. BK20171265) and the Foundation of High Level Health Talents of 'Six One' Project in Jiangsu Province (grant no. LGY2019048).

\section{Availability of data and materials}

The datasets used and/or analyzed during the current study are available from the corresponding author on reasonable request.

\section{Authors' contributions}

JY and QZ conceived and designed the study. JY, TF and MZ performed the experiments. JY and QZ analyzed the data. JY wrote the manuscript. QZ edited the manuscript. All authors read and approved the final manuscript.

\section{Ethics approval and consent to participate}

The study protocol was approved by the Ethics Committee of The Affiliated Huaian Hospital of Xuzhou Medical University (Huaian, China). Written informed consent was obtained from all patients.

\section{Patient consent for publication}

Not applicable.

\section{Competing interests}

The authors declare that they have no competing interests.

\section{References}

1. Mirabello L, Troisi RJ and Savage SA: Osteosarcoma incidence and survival rates from 1973 to 2004: Data from the surveillance, epidemiology, and end results program. Cancer 115: 1531-1543, 2009.

2. Wycislo KL and Fan TM: The immunotherapy of canine osteosarcoma: A historical and systematic review. J Vet Intern Med 29: 759-769, 2015.

3. Morris CD, Teot LA, Bernstein ML, Marina N, Krailo MD, Villaluna D, Janeway KA, DuBois SG, Gorlick RG and Randall RL: Assessment of extent of surgical resection of primary high-grade osteosarcoma by treating institutions: A report from the children's oncology group. J Surg Oncol 113: 351-354, 2016.

4. Xie X, Li YS, Xiao WF, Deng ZH, He HB, Liu Q and Luo W: MicroRNA-379 inhibits the proliferation, migration and invasion of human osteosarcoma cells by targetting EIF4G2. Biosci Rep 37: BSR20160542, 2017.

5. Zhang Y, He Z, Li Y, Yang Y, Shi J, Liu X, Yuan T, Xia J, Li D, Zhang $\mathrm{J}$ and Yang $\mathrm{Z}$ : Selection of surgical methods in the treatment of upper tibia osteosarcoma and prognostic analysis. Oncol Res Treat 40: 528-532, 2017.

6. Lee JA, Paik EK, Seo J, Kim DH, Lim JS, Yoo JY and Kim MS: Radiotherapy and gemcitabine-docetaxel chemotherapy in children and adolescents with unresectable recurrent or refractory osteosarcoma. Jpn J Clin Oncol 46: 138-143, 2016.

7. Ferrari S and Serra M: An update on chemotherapy for osteosarcoma. Expert Opin Pharmacother 16: 2727-2736, 2015.

8. Geller DS and Gorlick R: Osteosarcoma: A review of diagnosis, management, and treatment strategies. Clin Adv Hematol Oncol 8: 705-718, 2010.

9. Adamopoulos C, Gargalionis AN, Basdra EK and Papavassiliou AG: Deciphering signaling networks in osteosarcoma pathobiology. Exp Biol Med (Maywood) 241: 1296-1305, 2016.

10. Nagano $T$ and Fraser P: No-nonsense functions for long noncoding RNAs. Cell 145: 178-181, 2011.

11. Tehrani SS, Karimian A, Parsian H, Majidinia M and Yousefi B: Multiple functions of long non-coding RNAs in oxidative stress, DNA damage response and cancer progression. J Cell Biochem 119: 223-236, 2018.

12. Wilusz JE, Sunwoo H and Spector DL: Long noncoding RNAs: Functional surprises from the RNA world. Genes Dev 23: 1494-1504, 2009.

13. Ju C, Zhou R, Sun J, Zhang F, Tang X, Chen KK, Zhao J, Lan X, Lin S, Zhang Z and Lv XB: LncRNA SNHG5 promotes the progression of osteosarcoma by sponging the miR-212-3p/SGK3 axis. Cancer Cell Int 18: 141, 2018.

14. Zhang R, Liu Y, Liu H, Chen W, Fan HN, Zhang J and Zhu JS: The long non-coding RNA SNHG12 promotes gastric cancer by activating the phosphatidylinositol 3-kinase/AKT pathway. Aging (Albany NY) 11: 10902-10922, 2019.

15. Xia P, Liu P, Fu Q, Liu C, Luo Q, Zhang X, Cheng L, Qin T and Zhang H: Long noncoding RNA EPIC1 interacts with YAP1 to regulate the cell cycle and promote the growth of pancreatic cancer cells. Biochem Biophys Res Commun 522: 978-985, 2020.

16. Ding H, Luo Y, Hu K, Liu P and Xiong M: Linc00467 promotes lung adenocarcinoma proliferation via sponging miR-20b-5p to activate CCND1 expression. Onco Targets Ther 12: 6733-6743, 2019. 
17. Li GC, Xin L, Wang YS and Chen Y: Long intervening noncoding 00467 RNA contributes to tumorigenesis by acting as a competing endogenous RNA against miR-107 in cervical cancer cells. Am J Pathol 189: 2293-2310, 2019.

18. Livak KJ and Schmittgen TD: Analysis of relative gene expression data using real-time quantitative PCR and the 2(-Delta Delta C(T)) method. Methods 25: 402-408, 2001.

19. Majid S, Dar AA, Saini S, Yamamura S, Hirata H, Tanaka Y, Deng $G$ and Dahiya R: MicroRNA-205-directed transcriptional activation of tumor suppressor genes in prostate cancer. Cancer 116: 5637-5649, 2010

20. Yang Z, Chen J, Xie H, Liu T, Chen Y, Ma Z, Pei X, Yang W and Li L: Androgen receptor suppresses prostate cancer metastasis but promotes bladder cancer metastasis via differentially altering miRNA525-5p/SLPI-mediated vasculogenic mimicry formation. Cancer Lett 473: 118-129, 2020.

21. Chen J, Wu Z and Zhang Y: LncRNA SNHG3 promotes cell growth by sponging miR-196a-5p and indicates the poor survival in osteosarcoma. Int J Immunopathol Pharmacol 33: $2058738418820743,2019$.

22. Li G and Zhu Y: Effect of lncRNA ANRIL knockdown on proliferation and cisplatin chemoresistance of osteosarcoma cells in vitro. Pathol Res Pract 215: 931-938, 2019.

23. Su P, Mu S and Wang Z: Long noncoding RNA SNHG16 promotes osteosarcoma cells migration and invasion via sponging miRNA-340. DNA Cell Biol 38: 170-175, 2019.

24. Liu B, Zhao H, Zhang L and Shi X: Silencing of long-non-coding RNA ANCR suppresses the migration and invasion of osteosarcoma cells by activating the p38MAPK signalling pathway. BMC Cancer 19: 1112, 2019.

25. Song K, Yuan X, Li G, Ma M and Sun J: Long noncoding RNA CASC11 promotes osteosarcoma metastasis by suppressing degradation of snail mRNA. Am J Cancer Res 9: 300-311, 2019.

26. Li Z, Liu J, Chen H, Zhang Y, Shi H, Huang L, Tao J, Shen R and Wang T: Ferritin light chain (FTL) competes with long noncoding RNA Linc00467 for miR-133b binding site to regulate chemoresistance and metastasis of colorectal cancer. Carcinogenesis 41 : 467-477, 2019

27. Jiang W, Cheng X, Wang T, Song X, Zheng Y and Wang L: LINC00467 promotes cell proliferation and metastasis by binding with IGF2BP3 to enhance the mRNA stability of TRAF5 in hepatocellular carcinoma. J Gene Med 22: e3134, 2020.

28. D'Angelo B, Benedetti E, Cimini A and Giordano A: MicroRNAs: A puzzling tool in cancer diagnostics and therapy. Anticancer Res 36: 5571-5575, 2016.
29. Srivastava SK, Bhardwaj A, Leavesley SJ, Grizzle WE, Singh S and Singh AP: MicroRNAs as potential clinical biomarkers: Emerging approaches for their detection. Biotech Histochem 88 373-387, 2013 .

30. Cui Y, Yi L, Zhao JZ and Jiang YG: Long noncoding RNA HOXA11-AS functions as miRNA sponge to promote the glioma tumorigenesis through targeting miR-140-5p. DNA Cell Biol 36: 822-828, 2017

31. Li T, Meng XL and Yang WQ: Long noncoding RNA PVT1 Acts as a 'sponge' to inhibit microRNA-152 in gastric cancer cells. Dig Dis Sci 62: 3021-3028, 2017.

32. Yu H, Xu Y, Zhang D and Liu G: Long noncoding RNA LUCAT1 promotes malignancy of ovarian cancer through regulation of miR-612/HOXA13 pathway. Biochem Biophys Res Commun 503: 2095-2100, 2018.

33. Long B, Li N, Xu XX, Li XX, Xu XJ, Liu JY and Wu ZH: Long noncoding RNA LOXL1-AS1 regulates prostate cancer cell proliferation and cell cycle progression through miR-541-3p and CCND1. Biochem Biophys Res Commun 505: 561-568, 2018.

34. Jiang B, Zhu SJ, Xiao SS and Xue M: MiR-217 inhibits M2-like macrophage polarization by suppressing secretion of interleukin-6 in ovarian cancer. Inflammation 42: 1517-1529, 2019.

35. Yin Z and Ren W: MicroRNA-217 acts as a tumor suppressor and correlates with the chemoresistance of cervical carcinoma to cisplatin. Onco Targets Ther 12: 759-771, 2019.

36. Chen G, Yang Z, Feng M and Wang Z: microRNA-217 suppressed epithelial-to-mesenchymal transition through targeting PTPN14 in gastric cancer. Biosci Rep 40: BSR20193176, 2020.

37. Zhu L, Yang S and Wang J: miR-217 inhibits the migration and invasion of HeLa cells through modulating MAPK1. Int J Mol Med 44: 1824-1832, 2019.

38. Yang J, Lu C, Wei J, Guo Y, Liu W, Luo L, Fisch G and Li X: Inhibition of KPNA4 attenuates prostate cancer metastasis. Oncogene 36: 2868-2878, 2017

39. Li X, Yu M and Yang C: YY1-mediated overexpression of long noncoding RNA MCM3AP-AS1 accelerates angiogenesis and progression in lung cancer by targeting miR-340-5p/KPNA4 axis. J Cell Biochem 121: 2258-2267, 2020.

40. Zhang M, Luo H and Hui L: MiR-3619-5p hampers proliferation and cisplatin resistance in cutaneous squamous-cell carcinoma via KPNA4. Biochem Biophys Res Commun 513: 419-425, 2019. 